\begin{tabular}{|c|l|}
\hline Title & Lamprey Contractile Protein Genes Mark Different Populations of Skeletal Muscles During Development \\
\hline Author(s) & Kusakabe, Rie; Takechi, Masaki; Tochinai, Shin; Kuratani, Shigeru \\
\hline Citation & $\begin{array}{l}\text { Journal of Experimental Zoology Part B: Molecular and Developmental Evolution, 302B, 121-133 } \\
\text { https:/doi.org/10.1002/ez.b.20009 }\end{array}$ \\
\hline Issue Date & 2004-03-24 \\
\hline Doc URL & http://hdl.handle.net/2115/547 \\
\hline Rights & Copyright (c) 2004 Wiley-Liss, Inc. \\
\hline Type & article (author version) \\
\hline File Information & JEZ302B.pdf \\
\hline
\end{tabular}

Instructions for use 


\title{
Lamprey Contractile Protein Genes Mark Different Populations of Skeletal Muscles During Development
}

\author{
RIE KUSAKABE ${ }^{1, n}$, MASAKI TAKECHI $^{2,3}$, SHIN TOCHINAI $^{3}$, and SHIGERU KURATANI ${ }^{1}$ \\ ${ }^{1}$ Laboratory for Evolutionary Morphology, Center for Developmental Biology, \\ RIKEN, Japan \\ ${ }^{2}$ Department of Integrated Biosciences, Graduate School of Frontier Sciences, \\ The University of Tokyo, Tokyo, Japan \\ ${ }^{3}$ Division of Biological Sciences, Graduate School of Science, Hokkaido \\ University, Hokkaido, Japan
}

\begin{abstract}
Agnathan lampreys retain ancestral characteristics of vertebrates in the morphology of skeletal muscles derived from two mesodermal regions: trunk myotomes and unsegmented head mesoderm. During lamprey development, some populations of myoblasts migrate via pathways that differ from those of gnathostomes. To investigate the evolution of skeletal muscle differentiation in vertebrates, we characterize multiple contractile protein genes expressed in the muscle cells of the Japanese lamprey, Lethenteron japonicum. Lamprey actin gene LjMA2, and myosin heavy chain $(\mathrm{MyHC})$ genes $\mathrm{LjMyHC1}$ and $\mathrm{LjMyHC} 2$ are all expressed in the developing skeletal muscle cells of early embryos. However, LjMyHC1 and LjMyHC2 are expressed only in cells originating from myotomes, while LjMA2 is expressed in both myotomal and head musculature. Thus, in lampreys, myotomes and head mesoderm differ in the use of genes encoding contractile protein isoforms. Phylogenetic tree analyses including lamprey MyHCs suggest that the variety of muscle MyHC isoforms in different skeletal muscles may correspond to the morphological complexity of skeletal muscles of different vertebrate species. Another lamprey actin gene LjMA1 is likely to be the first smooth muscle actin gene isolated from non-tetrapods. We conclude that, in vertebrate evolution, the different regulatory systems for striated and smooth muscle-specific genes may have been established before the agnathan/gnathostome divergence. J. Exp. Zool. (Mol. Dev. Evol.) 302B:121-133, 2004.
\end{abstract}

\section{INTRODUCTION}

The morphological variety and specialized functions of skeletal muscles provide vertebrates with highly organized body movements. The developmental processes of the skeletal muscles of birds and mammals have long been studied (Dietrich, '99; Noden et al., '99; Buckingham, 2001). All the skeletal 
muscles except the pharyngeal and extraocular muscles in the head develop from segmental myotomes. In gnathostomes, myotomes split into epaxial and hypaxial muscles, separated by the horizontal septum, and innervated by dorsal (epaxial) and ventral (hypaxial) rami of the spinal nerves, respectively (Spo"rle, 2001). Some of the hypaxial myotomal cells undergo epithelialization and migrate a long distance to the location where they differentiate into multinuclear skeletal muscle cells. These migratory muscle cells give rise to the appendicular muscles, diaphragm, tongue, and trapezius (cucullaris) muscles in mammals (reviewed by Kuratani et al., 2002).

Although the basic body plan of agnathans does not differ from that of gnathostomes (e.g., dorsal spinal cord, brain, notochord, vertebrae, cranium, and pharyngeal arches), there is a large difference between the skeletal muscle patterning of the two animal groups. Lamprey skeletal muscles do not exhibit epaxial/hypaxial differentiation, and migratory cell lineages have not been observed to generate appendicular and other muscles as in gnathostomes. Thus, the patterning mechanism of the skeletal muscles appears to have undergone a dramatic change during the transition from agnathan to gnathostome. In this study, we focus on the patterns of expression of muscle marker genes of agnathans and gnathostomes to obtain insights into the genetic change required to produce the gnathostome-specific developmental program. We selected actin and myosin heavy chain (MyHC) genes, known to be differentially expressed in different vertebrate muscle tissues, as muscle-specific markers suitable for our analysis.

In mammals, four muscle actin genes (a-skeletal, a-cardiac, a-vascular, and g-enteric) are differentially regulated in skeletal, cardiac, and smooth muscle cells (Vandekerckhove and Weber, '79). Vertebrate muscle actins are more closely related to each other than to non-muscle actins, another group of actins that plays important roles in general cellular functions (see Fig. 2A). The actin gene family is unreported in lampreys, although partial amino acid sequences were analyzed and the existence of one muscle actin type has been predicted (Vandekerckhove and Weber, '84). Actin genes have been extensively studied in protochordates including ascidians (reviewed in Kusakabe, '97) and amphioxus (Bovenschulte and Weber, '97; Suzuki and Satoh, 2000; Kusakabe et al., '97a, '99b). Ascidian and amphioxus muscle actins are closely related to the vertebrate muscle actins. In contrast, echinoderm muscle actins are more closely related to vertebrate non-muscle actins than they are to vertebrate muscle actins (Fig. 2A). It has been suggested that the vertebrate- type of muscle actins genes are one of the molecular-level synapomorphies shared by chordates.

The conventional MyHCs that assemble to form filaments in muscle and non-muscle cells belong to class II of the large family of MyHC motor proteins (Sellers, '99). Different class II MyHC isoforms function in muscle and non-muscle cells. In contrast to actins, smooth muscle MyHCs are more closely related to non-muscle MyHCs than to striated muscle MyHCs (Goodson and Spudich, '93). Humans have at least 16 class II MyHC genes, including two non-muscle, one smooth muscle, and 13 striated muscle-types MyHCs (Berg et al., 2001; Desjardins et al., 2002). Although a vast number of nucleotide sequences of animal MyHCs have been registered in the database, many of them are partial due to the extraordinarily large size of MyHC genes: usually about six kilobases for the coding region alone. There has been no report of lamprey MyHC in protein or nucleotide levels. In the previous studies, we reported the regulatory function of upstream regions of striated muscle actin genes of the teleost, medaka Olyzias latipes (Kusakabe et al., '99a). These regions can activate transcription in skeletal and cardiac muscle cells when introduced into Lethenteron japonicum embryos (Kusakabe et al., 2003). Our results showed that the transcriptional regulatory mechanisms of striated muscle-specific actin genes are conserved between the lamprey and the medaka. To compare the gene family structures and the patterns of expression of muscle-specific genes of lamprey and gnathostomes, we characterize multiple actin and MyHC genes from the lamprey. The results shed light on the evolution of differential usage of contractile protein genes in muscle cells of vertebrates.

\section{MATERIALS AND METHODS}




\section{Obtaining lamprey embryos}

Ripe adults of the Japanese lamprey L. japonicum were purchased from the Ebetsu Fishermen's Association (Ebetsu, Hokkaido, Japan) and maintained at 101C. Spawning was induced and fry were reared to the appropriate developmental stages at 161 in fresh water or in 10\% Steinberg's solution (Steinberg, '57). Developmental stages were defined according to the description of Tahara ('88) for $L$. reissneri, a species closely related to L. japonicum.

\section{RT-PCR and cDNA library screening}

Total RNA (1 mg) of stage $25 \mathrm{~L}$. japonicum embryos was used as the template to synthesize the first-strand cDNA using an oligo(dT) primer according to the manufacturer's protocol (Super- Script Preamplification System for First Strand cDNA synthesis, Life Technologies, Inc., Rockville, MD). Actin cDNA fragments were amplified from the first-strand cDNA by polymerase chain reaction (PCR) with oligonucleotide primers ACT-F2 (5o-AATTGGGATGATATGGAGAA-3o) and ACTR2 (5o-ATCCACATTTGTTGGAAKGT-3o; K . G or T) (Kusakabe et al., '97a). Actin gene fragments corresponding to amino acids 78-357 of actin proteins were amplified (according to the numbering system in Vandekerckhove and Weber, ' 84$)$. The PCR products were subcloned into pBluescript II SK(t) (Stratagene), and sequencing was carried out using the dideoxy chain termination procedure (Sanger et al., '77) with an ABI Prism 310 and 3100 DNA Sequencers (Perkin-Elmer, Foster City, CA). cDNA fragments encoding a cytoplasmic actin (LjCA1) and two different muscle actins (LjMA1 and LjMA2) were identified.

Using LjMA1 and LjMA2 fragments as the probe, a L. japonicum larval head cDNA library (Ogasawara et al., 2000) and stage 24-26 embryonic cDNA library were screened. The probe was labeled with digoxigenin (DIG)-dUTP using the DIG-High prime (Roche). The hybridization was performed in $50 \%$ formamide, $5 \times \mathrm{xSC}, 0.1 \%$ Nlauroylsarcosine, $0.02 \%$ SDS, and $2 \%$ blocking reagent (Roche) at $421 \mathrm{C}$. Washes were $2 \mathrm{xSSC}$ and $0.1 \% \mathrm{SDS}$, used twice for $15 \mathrm{~min}$ at $501 \mathrm{C}$. Detection was carried out with the DIG Nucleic Acid Detection Kit (Roche) according to the manufacturer's instructions. Positive clones were in vivo excised into pBluescript SK(-) (Stratagene) and sequenced as described above.

Multiple cDNA fragments with homology to vertebrate class II MyHC genes were found in the EST (expressed sequence tag) database of L. japonicum stage 24-26 embryonic cDNA plasmid library. For each myosin gene, plasmid clones containing the longest insert were selected and fully sequenced as described above. All lamprey gene sequences reported in this paper were registered in GenBank/EMBL/DDBJ under accession numbers shown in Figures 2A and 5A.

\section{Molecular phylogenetic analysis}

The L. japonicum actin and MyHC cDNA sequences were compared using the GenetyxMac program (version 11.0, Software Development) and deduced amino acid sequences were aligned with those of other animals and a plant using the Clustal W program (Thompson et al., '94). Phylogenetic trees were constructed using the neighborjoining (NJ) method (Saiou and Nei, '87). One thousand bootstrap analyses were performed for the phylogenetic analysis. Maximum-likelihood (ML) trees (Felsenstein, '81) were reconstructed by the quartet puzzling algorithm using the TREE-PUZZLE program (Strimmer and von Haeseker, '96). Reliability values for each internal branch expressed in percent how often the corresponding cluster was found among the 1000 intermediate trees. Previously published sequences were obtained from GenBank/EMBL/ DDBJ and used for amino acid comparison and phylogenetic analyses. The accession numbers for previously published sequences are indicated in Figures 2A and 5A.

\section{In situ hybridization}

Whole-mount in situ hybiridization of lamprey embryos was performed as described in Ogasawara et al. 
(2000). For actin genes, probes were designed to correspond exclusively to the 30 untranslated regions (UTR) of each gene to avoid cross-hybridization. For MyHC genes, probes contained the whole 3oUTR plus a 30 part of coding sequences. Each probe was between 200 and $500 \mathrm{bp}$ long.

\section{RESULTS}

\section{Characterization of lamprey actin cDNAs}

cDNA clones coding for three different actin genes were isolated from embryonic and larval cDNA libraries of L. japonicum. All three cDNAs contained parts of 5oUTR, full open reading frame (ORF), and 3oUTR. To elucidate the evolutionary relationships of actin isoforms of lamprey and other chordates, we compared isoform-specific amino acid residues (Fig. 1) and constructed phylogenetic trees (Fig. 2). The 21 amino acid residues shown in Figure 1 differ among mammalian a-skeletal and cytoplasmic actins (Vandekerckhove and Weber, '84). The amino acid position 89 was included, as it is diagnostic to smooth muscle isoforms. For chordates, actin proteins can be tentatively categorized to different isoforms by comparing amino acid residues at these positions (Kusakabe, '97; Kovilur et al., '93; Kusakabe et al., '97a). The two lamprey actins that shared all, or 20, of these 21 residues with mammalian muscle actins were identified as being of muscle-type and named LjMA1and LjMA2, respectively. The other lamprey actin shared all of the 21 residues with mammalian non-muscle actins and was named LjCA1. The ratio of LjMA1 transcripts contained in the embryonic cDNA library was low. Of 19 positive clones obtained in our embryonic cDNA library screening using the LjMA1 fragment as the probe, only one corresponded to LjMA1 and the rest to LjMA2.

Full amino acid sequences of actin proteins were used to produce the phylogenetic tree using the NJ method (Fig. 2A). The node closest to the outgroup Arabidopsis thaliana actin tied two large clusters: one exclusively consisting of chordate muscle actins and the other of non-muscle actins plus non-chordate muscle actins. Consistent with the results of the comparison of amino acid residues described above, LjCA1 was grouped with the vertebrate non-muscle actins, whereas LjMA1 and LjMA2 were placed in the chordate muscle actin clade. Within the muscle clade, LjMA1 was closely related to vertebrate smooth muscle actins, whereas LjMA2 was grouped with vertebrate striated muscle actins at a low bootstrap value. Consistently, at amino acid positions 17 and 89, LjMA1 had residues found in the mammalian smooth muscle actins, but not in striated muscle actins (Fig. 1).

At amino acid position 17, LjMA2 possessed a Cys residue that is common in mammalian smooth muscle- and non-muscle actins, rather than a Val shared by the striated muscle actins of vertebrates (Vandekerckhove and Weber, '79), ascidians (Kusakabe, '97; Chiba et al., 2003), appendicularia (Nishino et al., 2000), and amphioxus (Kusakabe et al., '97a; Suzuki and Satoh, 2000). The Val at position 17 may have existed in the ancestral protein of chordate striated muscle actins, and been substituted by a Cys residue independently in the lamprey striated actin lineage. The grouping of LjMA2 with human and medaka (OlMA1 and OlMA2) striated muscle actins was supported by a low bootstrap value (around 20\%). The monophyly of human and medaka striated muscle actins is supported by a relatively high bootstrap value (higher than 70\%; data not shown; Kusakabe et al., '99a). Thus, the low bootstrap value at the node of vertebrate striated muscle actin cluster (189/1000) appears to be due to the addition of LjMA2. To confirm the reliability of $\mathrm{NJ}$ tree, we carried out an additional phylogenetic analysis using the ML method (Fig. 2B). Again in this analysis, LjCA1 and LjMA1 were most closely related to vertebrate cytoplasmic and smooth muscle actins, respectively. However, unlike in NJ tree, LjMA2 was also grouped with vertebrate smooth muscle actins with a low reliability value $(59 \%)$. Therefore, LjMA2 amino acid sequence contains both striated and smooth muscle-like characteristics, although its expression pattern strongly suggests that it is categorized as a striated muscle actin (see below).

\section{Expression of lamprey actin genes throughout development}


Expression patterns of LjMA1 and LjMA2 were analyzed by whole-mount in situ hybridization. As expected from the phylogenetic tree (Fig. 2A), LjMA2 was expressed exclusively in the skeletal and cardiac muscles (Fig. 3). This gene was not expressed earlier than stage 21, although somites have started to form by this stage (Fig. 3A). Expression of LjMA2 started in several anterior somites at stage 22 (Fig. 3B), and spread gradually to the caudal somites (Fig. 3C). At stage 24 (Fig. 3D), when the rostralmost myotomes start to split dorsoventrally, a faint expression was seen in the upper lip. These cells correspond to the primordium of the upper lip muscle already described in the literature (Sewertzoff, '16; Holland et al., '93; Kuratani et al., '97, 2001). Subsequently, LjMA2 was expressed in the lower lip, as well as in more posteriorly located pharyngeal arch muscles (Fig. 3E). Rostrally extending supra and infraoptic muscles, the myotomal muscles characteristic to lampreys (see discussion), expressed LjMA2 (Fig. 3E). At stage 25, the heart also started to express LjMA2 (Fig. 3E). At stage 28, all the skeletal musculatures, including oral, velar, and other pharyngeal muscles, were marked by the expression of LjMA2 (Fig. 3F). At the anmocoetes larval stage (Fig. 3G), LjMA2 expression in the trunk region was faint, because the LjMA2-positive myotomal musculature extends ventrally and reduces in thickness to surround the gut. At this stage, LjMA2 was also expressed in the hypobranchial muscles, which are derived from the anterior myotomes. These muscles correspond to the tongue muscles of the gnathostomes, which take a distinctive migratory pathway from the anterior myotomes (Kuratani et al., '98, 2002; see discussion).

Expression of another muscle actin gene, LjMA1, started in the cheek process of stage 23 embryos (Fig. 4A). The mesodermal layer of this region probably raises skeletal muscles of the oral region (Kuratani et al., 2001). This early expression may mark the skeletal muscle precursor cells. At later stages, the upper lip muscles continuously expressed LjMA1 (Figs. 4B, D). At stage 28, a strong and specific LjMA1 expression was seen in the digestive tract (esophagus) and the oral region (Figs. 4B, C). Expression was also observed at the midline, dorsal to the developing gut along the rostrocaudal axis (Fig. 4C). This may mark the presumptive dorsal aorta. Expression in the digestive tract spread caudally to the broad part of the gut of stage 30 embryos (Fig. 4E). In our experiment, the breadth of the LjMA1-positive area of the gut varied in each individual. LjMA1 expression was not observed in the myotomal skeletal muscles. Thus, it is inferred that LjMA1 is the muscle actin gene with strong specificity for smooth muscle and for a part of skeletal muscle tissues of the head. Consistent with the low number of LjMA1 clones obtained from the cDNA library, the expression of this gene was generally much weaker than that of LjMA2 through all the developmental stages examined.

The non-muscle actin gene, LjCA1, was ubiquitously expressed in the whole body (data not shown), except for the developing myotomes in which LjMA2 was expressed at high levels. It is known that non-muscle actin isoforms of gnathostomes are downregulated in muscle cells, and a regulatory sequence involved in this downregulation has been identified in the mammalian b-actin gene (DePonti-Zilli et al., '88). Thus, the regulatory mechanism for non-muscle actin genes might be conserved between agnathans and gnathostomes.

\section{Characterization of the lamprey myosin heavy chain cDNAs}

We found multiple cDNA clones encoding MyHC proteins in the EST database of a stage $24 \mathrm{~L}$. japonicum embryonic cDNA library. Among them, four different class II myosin heavy chain genes were identified and named LjMyHC1 through LjMyHC4. LjMyHC1 was identified as a cDNA clone containing the complete MyHC ORF. The other three LjMyHC genes were found as partial clones, lacking various lengths of 5 'parts of ORFs. LjMyHC2 lacked the N-terminal sequence of the head domain and contained the complete rod domain. LjMyHC1 and LjMyHC2, both of which are of skeletal muscle-type (see below), had a short 3'UTR sequence of about $110 \mathrm{bp}$. However, no apparent similarity was found between these 3'UTR sequences. Both LjMyHC3 and LjMyHC4, for which only the 3'termini of C-terminal rod domain plus 3'UTRs were obtained, seemed to represent non-muscle isoforms, as suggested by our preliminary phylogenetic analysis (data not shown).

To estimate the functions and phylogenetic positions of lamprey MyHCs, deduced amino acid sequences 
of C-terminal rod domains of LjMyHC1 and LjMyHC2 were compared with selected human and Ciona MyHCs using the NJ method (Fig. 5A). The two lamprey MyHCs formed an exclusive cluster, which was most closely related to vertebrate fast skeletal muscle MyHCs. However, the cluster of LjMyHC1 and LjMyHC2 branched outside of all human fast skeletal MyHCs (IIa, IIb, IIx, and developmental isoforms). No lamprey isoform was found to correspond to any human fast skeletal MyHC. The same result was obtained from another tree constructed using the ML method (Fig. 5B). In birds and mammals, multiple slow and fast MyHCs are differentially expressed in each muscle (reviewed in Wigmore and Evans, 2002). It has also been reported that the bovine skeletal MyHC content of tongue and diaphragm, both of which are absent from lampreys, differs greatly from that in other skeletal muscles (see Kuratani et al., '97 for review of muscle morphology; Tanabe et al., '98). Thus, the differential usage of fast skeletal myosin isoforms might be in parallel with the evolution of the complex morphology of the skeletal muscles of gnathostomes. Results from the phylogenetic analysis of the MyHCs provide new insights into protochordate MyHC evolution. Ascidian C. intestinalis genome contains six MyHC genes, among which Ci-MHC5 is expressed in the segmented tail muscles (Chiba et al., 2003). This gene represents an isoform most closely related to vertebrate striated muscle MyHCs (Fig. 5). An MyHC that is specific to the adult body wall muscle (Ci-MHC3) is placed outside all the chordate striated muscle MyHCs. The ascidian body wall muscle consists of smooth muscle cells (Terakado and Obinata, '87), but the major structural proteins employed by these cells are of the striated muscle-type (Meedel and Hastings, '93; Endo et al., '96). Thus, ascidian muscle MyHCs includes two types: those closely related to the vertebrate striated muscle MyHCs expressed in embryonic striated locomotory muscles, such as Ci-MHC5, and those that diverged early in evolution and are expressed in smooth muscle cells exhibiting some of the molecular characteristics of striated muscles, such as $\mathrm{Ci}-\mathrm{MHC}$. None of the six Ciona MyHCs is clustered inside the vertebrate fast skeletal/cardiac MyHC clade (Fig. 5). These results support the view that multiplied MyHCs expressed in vertebrate skeletal muscle may have evolved subsequent to the emergence of craniates (see discussion).

\section{Expression of lamprey myosin heavy chain genes during lamprey development}

The patterns of expression of LjMyHC1 and LjMyHC2 were analyzed by whole mount in situ hybridization. In the present study, only the $\mathrm{LjMyHC1}$ expression pattern is presented, since $\mathrm{LjMyHC1}$ and LjMyHC2 are expressed in almost identical spatiotemporal patterns (Fig. 6). At stage 23, LjMyHC1 was specifically expressed in developing myotomes (Fig. 6A). As development proceeded, myotomal expression of LjMyHC1 became stronger (Fig. 6B). Significantly, no expression of LjMyHC1 was observed in skeletal muscles originating from the head mesoderm (Fig. 6C). At stage 29, when LjMA2 was expressed in many of the muscles derived from the head mesoderm (Fig. 3F), LjMyHC1 was expressed only in the trunk myotomes, including those that grew rostrally to differentiate into the infraand supraoptic muscles (Fig. 6D). At stage 30, LjMyHC1 was expressed in the ventrally extending trunk skeletal muscle (Fig. 6E), supra- and infraoptic muscles (Fig. 6F), and in the hypobranchial muscle (see discussion). Thus, $\mathrm{LjMyHC1}$ and $\mathrm{LjMyHC2}$ were expressed exclusively in myotomal skeletal muscles throughout development.

\section{DISCUSSION}

We characterized multiple cDNAs encoding contractile proteins in the lamprey, L. japonicum. We discussed our analyses from both phylogenetic and developmental points of view. The lamprey genes reported here may reflect the dual nature of this animal, both as a key animal positioned between protochordates and extant gnathostomes, and as a highly evolved agnathan species with a uniquely organized gene family structure and body plan.

\section{Molecular phylogeny of actins and MyHCs}

In Figure 7 we summarize the evolutionary history of chordate actin and MyHC genes inferred from this 
and previous studies (Vandekerckhove and Weber, '84; Oota and Saitou, '99; Kovilur et al., '93; Kusakabe et al., '97b). One of the major new insights reflected in this scheme is that lampreys have already acquired a smooth muscle isoform. Vandekerckhove and Weber ('84) predicted the existence of a single actin gene expressed in the skeletal musculature of the lamprey L. fluviatilis on the basis of direct sequencing analysis of proteolytic peptides of actin proteins. In their analyses, smooth muscle-specific actin was not detected, which might probably be caused by the limited amount of protein product of this isoform. They concluded that the smooth muscle actin genes might have appeared after the divergence of chondrichthyes (Vandekerckhove and Weber, '84). In this study, the lamprey actin gene LjMA1 was discovered as a putative smooth muscle actin isoform. Thus, an ancestral type of vertebrate smooth muscle actin seems to have evolved subsequent to the agnathan divergence from the protochordate ancestors, but before the agnathan/gnathostome divergence (Fig. 7).

Lampreys have invented MyHCs that are expressed in subpopulations of the skeletal muscles, but not in the cardiac muscle. Expressions of $\mathrm{LjMyHC1}$ and $\mathrm{LjMyHC} 2$ were restricted to the somitic skeletal muscles, suggesting the existence of other MyHCs expressed in the head mesoderm. It is evident that lamprey somites and head mesoderms employ different regulatory mechanisms for MyHC genes. In addition, lamprey may possess a yet undiscovered MyHC gene that is expressed in the cardiac muscle.

\section{Duplication of contractile protein genes and body plan innovation}

Divergence of the multiple isoforms of actins and MyHCs is not synchronous through evolution (Fig. 7). Actins for all four types of vertebrate muscles are closely related to each other and seem to have appeared following the divergence of vertebrates. This contrasts with striated and smooth muscle MyHCs that evolved independently from non-muscle MyHCs. Therefore, the evolution of animal muscle tissues may not be inferred directly from the phylogenetic data of structural genes, even by integrating multiple gene trees, as suggested by Oota and Saitou ('99). One reason for this might lie in the fact that genes encoding relatively similar proteins are expressed differently in various types of muscles. Recent reports of genome-wide surveys of ascidian Ciona intestinalis muscle structural protein genes revealed that this animal contains 14 actin genes, consisting of six muscle-type and eight non-muscle-type genes, but no smooth muscle-type actin gene (Chiba et al., 2003). Another ascidian species Halocynthia roretzi has tandemly linked muscle actin genes whose tail muscle-specific expression patterns resemble each other (Kusakabe, '97; Kusakabe et al., '95). In this case, gene duplications seem to have occurred independently in several ascidian lineages, and the paralogues have not been subfunctionalized, but retain similar expression patterns. This type of gene duplications may allow rapid accumulation of gene products during the extremely accelerated development of ascidians, although they might not directly lead to a new body plan. In contrast, correlations can be found between muscle complexity and gene duplications for mammalian muscle actin isoforms. These isoforms are highly subfunctionalized, each specifically expressed in a single muscle cell type. Although the cardiac isoform is expressed both in cardiac and skeletal muscles early in development, its role is taken over by the skeletal isoform later in development (Buckingham, '92). Another example is multiple fast skeletal muscle MyHCs in the gnathostomes, which probably have appeared after the divergence of craniates, as mentioned above.

The regulatory regions of duplicated structural genes have undergone recent nucleotide substitutions that may provide a critical change in the patterns of expression of the downstream genes. Among vertebrate actins and MyHCs, lamprey cognates are of particular interest, since changes in gene expression patterns may reflect innovations in muscle tissues and body plan.

\section{Characteristics of lamprey skeletalmusculature as visualized by actin and MyHC gene expression}

Lamprey skeletal muscles have been visualized histochemically using AchE activity and immunochemically using $\mathrm{CH}-1$ tropomyosin antibody (Kuratani et al., '97, '99). These methods have revealed that lampreys do possess hypobranchial muscle that is probably homologous to the 
gnathostome tongue muscle derived from somites at the occipital level. Precursors of the lamprey hypobranchial muscle originate from several anterior somites and migrate ventrally along the caudal aspect of the pharyngeal arches (Neal, 1897; Kuratani et al., '97). These cells reach the lateral aspect of the pharyngeal wall where they undergo segmentation and start to express the above-mentioned marker genes. Moreover, lamprey hypobranchial muscle is innervated by the hypoglossal nerve, as is the case in gnathostome tongue muscles.

In this study, lamprey actin genes (LjMA2) and MyHC genes ( $\mathrm{LjMyHC1}$ and $\mathrm{LjMyHC} 2)$ were found to serve as effective skeletal muscle markers in visualizing lamprey muscle cells throughout development. Specifically, the pattern of expression of LjMA2 in the head region (Figs. 3D-G) illustrates the order of muscle formation from head mesoderm. The first head muscle differentiates in the upper lip, followed by the lower lip muscle that locates medially to the upper lip muscle. LjMA2 expression then spreads to the velum and caudal branchial arches in an anteriorto- posterior direction, staining adductor and constrictor muscles at each arch. Finally, the hypobranchial muscle, which arrives via the rostroventral pathway from occipital somites, expresses LjMA2 in a segmented pattern.

The segmentation patterns of epibranchial and hypobranchial muscles of the lampreys apparently correspond to the locations of gill pores, and they have sometimes been mistaken as an evidence for the head mesoderm segmentation, a situation similar to the anterior somites of amphioxus (Neal, 1897; Neal and Rand, '46). Expression of LjMyHC1 is absent in head mesoderm derivatives, and clearly visualizes specifically the trunk myotome derivatives (Fig. 6). Anterior myotomes, already expressing LjMyHC1, split dorsoventrally and extend rostrally to form supra- and infraoptic muscles. This is in clear contrast to the abovementioned hypobranchial muscle, which undergoes muscle differentiation after it has completed migration, as indicated by the retarded commencement of LjMA2 and LjMyHC1 expression. Thus, the epibranchial muscle of the lamprey is nothing more than segmented anterior myotomes that shift anteriorly to cover the head region, whereas the segmentation in hypobranchial muscle is acquired secondarily in a mass of undifferentiated myoblasts. In mammals, visualization of skeletal muscle precursors in limbs and tongue has been achieved using molecular probes such as the Lbx1 gene, an upstream regulator for the migratory lineage of hypaxial muscles (Dietrich et al., '99). Other members of the gene cascade regulating migration, such as Pax-3, c-met, and HGF, have been extensively studied (reviewed by Birchmeier and Brohmann, 2000). Characterization of these regulatory genes and their expression patterns in lamprey should provide us with information on the evolutionary pathway of complex skeletal muscle morphology in extant vertebrates.

\section{ACKNOWLEDGMENTS}

We thank Dr. Michio Ogasawara at Chiba University for providing us with the lamprey head cDNA library, Dr. Takehiro Kusakabe at Himeji Institute of Technology for advice on the molecular phylogenetic analyses, and Drs. Yasunori Murakami and Shigehiro Kuraku at CDB for the lamprey EST project.

\section{LITERATURE CITED}

Berg JS, Powell BC, Cheney RE. 2001. A millennial myosin census. Mol Biol Cell 12:780-794. Birchmeier C, Brohmann H. 2000. Genes that control the development of migrating muscle precursor cells. Curr Opin Cell Biol 12:725-730.

Bovenschulte M, Weber K. 1997. Deuterostomic actin genes and the definition of the chordates: cDNA cloning and gene organization for cephalochordates and hemichordates. J Mol Evol 45:653-660.

Buckingham M. 1992. Making muscle in mammals. Trends Genet 8:144-149.

Buckingham M. 2001. Skeletal muscle formation in vertebrates. Curr Opin Genet Dev 11:440-448.

Chiba S, Awazu S, Itoh M, Chin-Bow ST, Satoh N, Satou Y, Hastings KEM. 2003. A genomewide survey of developmentally relevant genes in Ciona intestinalis. IX. Genes for muscle structural proteins. Dev Genes Evol 213:291-302.

DePonti-Zilli L, Seiler-Tuyns A, Paterson BM. 1988. A 40-base-pair sequence in the 30 end of the b-actin gene regulates b-actin mRNA transcription during myogenesis. Proc Natl Acad Sci USA 85:1389-1393. 
Desjardins PR, Burkman JM, Shrager JB, Allmond LA, Stedman HH. 2002. Evolutionary implications of three novel members of the human sarcomeric myosin heavy chain gene family. Mol Biol Evol 19:375-393.

Dietrich S. 1999. Regulation of hypaxial muscle development. Cell Tissue Res 296:175-182.

Endo T, Matsumoto K, Hama T, Ohtsuka Y, Katsura G, Obinata T. 1996. Distinct troponin T genes are expressed in embryonic/larval tail striated muscle and adult body wall smooth muscle of ascidian. J Biol Chem 271: 27855-27862.

Felsenstein J. 1981. Evolutionary trees from DNA sequences: a maximum likelihood approach. J Mol Evol 17:368-376.

Goodson HV, Spudich JA. 1993. Molecular evolution of the myosin family: relationships derived from comparisons of amino acid sequences. Proc Natl Acad Sci USA 90:659-663.

Holland ND, Holland LZ, Honma Y, Fujii T. 1993. Engrailed expression during development of a lamprey, Lampetra japonica: a possible clue to homologies between agnathan and grathostome muscles of the mandibular arch. Dev Growth Differ 35:153-160. Kovilur S, Jacobson JW, Beach RL, Jeffery WR, Tomlinson CR. 1993. Evolution of the chordate muscle actin gene. J Mol Evol 36:361-368.

Kuratani S, Ueki T, Aizawa S, Hirano S. 1997. Peripheral development of cranial nerves in a cyclostome, Lampetra japonica: morphological distribution of nerve branches and the vertebrate body plan. J Comp Neurol 384:483-500.

Kuratani S, Horigome N, Ueki T, Aizawa S, Hirano S. 1998. Stereotyped axonal bundle formation and neuromeric patterns in embryos of a cyclostome, Lampetra japonica. J Comp Neurol 391:99-114.

Kuratani S, Horigome N, Hirano S. 1999. Developmental morphology of the head mesoderm and reevaluation of segmental theories of the vertebrate head: evidence from embryos of an agnathan vertebrate, Lampetra japonica. Dev Biol 210:381-400. Kuratani S, Nobusada Y, Horigome N, Shigetani Y. 2001. Embryology of the lamprey and evolution of the vertebrate jaw: insights from molecular and developmental perspectives. Phil Trans R Soc Lond B 356: 1615-1632.

Kuratani S, Kuraku S, Murakami Y. 2002. Lamprey as an evodevo model: lessons from comparative embryology and molecular phylogenetics. Genesis 34:175-183.

Kusakabe T. 1997. Ascidian actin genes: developmental regulation of gene expression and molecular evolution. Zool Sci $14: 707-718$.

Kusakabe T, Hikosaka A, Satoh N. 1995. Coexpression and promotor function in two muscle actin gene complexes of different structural organization in the ascidian Halocynthia roretzi. Dev Biol 169:461-472.

Kusakabe R, Kusakabe T, Satoh N, Holland ND, Holland LZ. 1997a. Differential gene expression and intracellular mRNA localization of amphioxus actin isoforms throughout development: implications for conserved mechanisms of chordate development. Dev Genes Evol 207:203-215.

Kusakabe R, Kusakabe T, Suzuki N. 1999a. In vivo analysis of two striated muscle actin promoters revealed combinations of multiple regulatory modules required for skeletal and cardiac muscle-specific gene expression. Int J Dev Biol 43:541-554.

Kusakabe R, Satoh N, Holland LZ, Kusakbe T. 1999b. Genomic organization and evolution of actin genes in the amphioxus Branchiostoma belcheri and Branchiostoma floridae. Gene 227:1-10.

Kusakabe R, Tochinai S, Kuratani S. 2003. Expression of foreign genes in lamprey embryos: an approach to study evolutionary changes in gene regulation. J Exp Zool (Mol Dev Evol) 296B:87-97.

Kusakabe T, Araki I, Satoh N, Jeffery WR. 1997b. Evolution of chordate actin genes: evidence from genomic organization and amino acid sequences. J Mol Evol 44:289-298.

Meedel TH, Hastings KEM. 1993. Striated muscle-type tropomyosin in a chordate smooth muscle, ascidian bodywall muscle. J Biol Chem 268:6755-6764.

Neal HV. 1897. The development of the hypoglossus musculature in Petromyzon and Squalus. Anat Anz 13: 441-463.

Neal HV, Rand HW. 1946. Comparative Anatomy. Philadelphia: Blakiston.

Nishino A, Satou Y, Morisawa M, Satoh N. 2000. Muscle actin genes and muscle cells in the appendicularian, Oikopleura longicauda: phylogenetic relationships among muscle tissues in the urochordates. J Exp Zool (Mol Dev Evol) 288:135-150. Noden DM, Marcucio R, Borycki A-G, Emerson Jr. CP. 1999. Differentiation of avian craniofacial muscles: I. Patterns of early regulatory gene expression and myosin heavy chain synthesis. Dev Dyn 216:96-112.

Ogasawara M, Shigetani Y, Hirano S, Satoh N, Kuratani S. 2000. Pax1/Pax9-related genes in an agnathan vertebrate, Lampetra japonica: expression pattern of LjPax9 implies sequential evolutionary events toward the gnathostome body plan. Dev Biol 223:399-410.

Oota S, Saitou N. 1999. Phylogenetic relationship of muscle tissues deduced from superimposition of gene trees. Mol Biol Evol $16: 856-867$.

Saitou N, Nei M. 1987. The neighbor-joining method: a new method for reconstructing phylogenetic trees. Mol Biol Evol 4:406-425.

Sanger F, Nicklen S, Coulson AR. 1977. DNA sequencing with chain-terminating inhibitors. Proc Natl Acad Sci USA 74:5463-5467. Sellers JR. 1999. Myosins. 2nd ed. New York: Oxford University Press.

Spo"rle R. 2001. Epaxial-adaxial-hypaxial regionalization of the vertebrate somite: evidence for a somitic organizer and a mirror-image duplication. Dev Genes Evol 211:198-217.

Steinberg M. 1957. A nonnutrient culture medium for amphibian embryonic tissues. Carnegie Institution of Washington Year Book 56:347-348.

Strimmer K, von Haeseler A. 1996. Quartet puzzling: a quartet maximum-likelihood method for reconstructing tree topologies. Mol Biol Evol 13:964-969.

Sewertzoff AN. 1916. Etudes sur l'e'volution des Verte'bre's inferieurs. I Arch Russ Anat Hisol Embryol I.

Suzuki MM, Satoh N. 2000. Genes expressed in the amphioxus notochord revealed by EST analysis. Dev Biol 224: 168-177. 
Tahara Y. 1988. Normal stages of development in the lamprey, Lampetra reissneri (Dybowski). Zool Sci 5: 109-118.

Tanabe R, Muroya S, Chikuni K. 1998. Sequencing of the 2a, 2x, and slow isoforms of the bovine myosin heavy chain and the different expression among muscles. Mam Genome 9:1056-1058.

Terakado K, Obinata T. 1987. Structure of multinucleated smooth muscle cells of the ascidian Halocynthia roretzi. Cell Tissue Res 247:85-94.

Thompson JD, Higgins DG, Gibson TJ. 1994. CLUSTAL W: improving the sensitivity of progressive multiple sequence alignment through sequence weighting, position specific gap penalties, and weight matrix choice. Nucleic Acid Res. 22:4673-4680.

Vandekerckhove J, Weber K. 1979. The complete amino acid sequence of actins from bovine aorta, bovine heart, bovine fast skeletal muscle, and rabbit slow skeletal muscle. A protein-chemical analysis of muscle actin differentiation. Differentiation 14:123-133.

Vandekerckhove J, Weber K. 1984. Chordate muscle actins differ distinctly from invertebrate muscle actins: the evolution of the different vertebrate muscle actins. J Mol Biol 179:391-413.

Wigmore PM, Evans DJR. 2002. Molecular and cellular mechanisms involved in the generation of fiber diversity during myogenesis. Int Rev Cytol 216:175-232. 


\begin{tabular}{|c|c|c|c|c|c|c|c|c|c|c|c|c|c|c|c|c|c|c|c|c|c|}
\hline & \multicolumn{21}{|c|}{ Amino acid poštion } \\
\hline & 5 & 6 & 10 & 16 & 17 & 76 & 89 & 103 & 129 & 153 & 162 & 176 & 201 & 225 & 260 & 267 & 272 & 279 & 287 & 297 & 365 \\
\hline Mammalian skeletel muscle attin & $\mathbf{T}$ & $\mathbf{T}$ & c & $\mathbf{L}$ & $\mathbf{v}$ & I & $\mathbf{T}$ & $\mathbf{T}$ & $\mathbf{v}$ & $\mathbf{L}$ & $\mathbf{N}$ & м & $\mathrm{v}$ & $\mathbf{N}$ & $\mathbf{T}$ & I & $\mathbf{A}$ & $\mathbf{Y}$ & I & $\mathbf{N}$ & $\mathbf{A}$ \\
\hline Mammulian candac muscle actin & $\mathbf{T}$ & $\mathbf{T}$ & c & $\mathbf{L}$ & $\mathbf{v}$ & I & $\mathbf{T}$ & $\mathbf{T}$ & $\mathbf{v}$ & $\mathbf{L}$ & $\mathbf{N}$ & м & $\mathbf{v}$ & $\mathbf{N}$ & $\mathbf{T}$ & I & A & $\mathbf{Y}$ & $\mathbf{I}$ & $\mathbf{N}$ & $\mathbf{A}$ \\
\hline Mamma lian esteric smoch maxcle atin & $\mathbf{T}$ & $\mathbf{T}$ & c & L & $\mathrm{c}$ & I & $\mathrm{s}$ & $\mathbf{T}$ & $\mathbf{v}$ & $\mathbf{L}$ & $\mathbf{N}$ & м & $\mathbf{v}$ & $\mathbf{N}$ & $\mathbf{T}$ & I & $\mathbf{A}$ & $\mathbf{Y}$ & I & $\mathbf{N}$ & $\mathbf{A}$ \\
\hline Mammalian acrta smooth muscle actin & $\mathbf{s}$ & $\mathbf{T}$ & c & $\mathbf{L}$ & $\mathrm{c}$ & $\mathbf{I}$ & $s$ & $\mathbf{T}$ & $\mathbf{v}$ & $\mathbf{L}$ & $\mathbf{N}$ & M & $\mathbf{v}$ & $\mathbf{N}$ & $\mathbf{T}$ & I & $A$ & $\mathbf{Y}$ & $\mathbf{I}$ & $\mathbf{N}$ & $\mathbf{A}$ \\
\hline Mammulian non-muscle betz-xtin & 1 & $\mathrm{~A}$ & $\mathrm{v}$ & M & $\mathrm{c}$ & $\mathrm{v}$ & $\mathbf{T}$ & $\mathrm{v}$ & $\mathrm{T}$ & M & $\mathrm{T}$ & $\mathrm{L}$ & $\mathrm{T}$ & Q & $\mathrm{A}$ & $\mathrm{L}$ & $\mathrm{c}$ & $\mathrm{F}$ & $\mathrm{v}$ & $\mathrm{T}$ & $\mathrm{s}$ \\
\hline Mammalian nan-muscle gamma-actin & 1 & $\mathrm{~A}$ & 1 & M & $\mathrm{c}$ & $\mathrm{v}$ & $\mathbf{T}$ & $\mathrm{v}$ & $\overline{\mathrm{T}}$ & $\mathrm{M}$ & $\mathrm{T}$ & $\mathrm{L}$ & $\mathrm{r}$ & Q & $\mathrm{A}$ & $\mathrm{L}$ & $\mathrm{c}$ & $\mathrm{F}$ & $\mathrm{v}$ & $\mathrm{T}$ & $\mathrm{s}$ \\
\hline LiMAI & $\mathbf{T}$ & $\mathbf{T}$ & c & $\mathbf{L}$ & $\mathrm{c}$ & $\mathbf{I}$ & $\mathrm{s}$ & $\mathbf{T}$ & $\mathbf{v}$ & $\mathbf{L}$ & $\mathbf{N}$ & м & $\mathbf{v}$ & $\mathbf{N}$ & $\mathbf{T}$ & I & $\mathbf{A}$ & $\mathbf{Y}$ & I & $\mathbf{N}$ & $\mathbf{A}$ \\
\hline L,MNA2 & $\mathbf{T}$ & $\mathbf{T}$ & c & L & $\mathrm{c}$ & I & $\mathbf{T}$ & $\mathbf{T}$ & $\mathbf{v}$ & L & $\mathbf{N}$ & $\mathbf{M}$ & $\mathbf{v}$ & $\mathbf{N}$ & $\mathbf{T}$ & I & $A$ & $\mathbf{Y}$ & I & $\mathbf{N}$ & $\mathbf{A}$ \\
\hline LijCAl & I & A & $\mathrm{v}$ & M & $\mathrm{c}$ & $\mathrm{v}$ & $\mathbf{T}$ & $\mathrm{v}$ & $\mathrm{T}$ & M & $\mathrm{T}$ & L & $\mathrm{T}$ & $Q$ & $\mathrm{~A}$ & L & c & $\mathrm{F}$ & $\mathrm{v}$ & $\mathrm{T}$ & $\mathrm{s}$ \\
\hline
\end{tabular}

Fig. 1. Comparison of diagnostic amino acid residues of L. japonicum actins with mammalian actins. Amino acid residues shared by the mammalian muscle actin proteins are shown in white letters in black boxes. Amino acid residues shared by the mammalian non-muscle actin proteins are shown in black letters in white boxes. Amino acid residue specific to mammalian smooth muscle actins (position 89) is shown in black letters in gray boxes. 
A

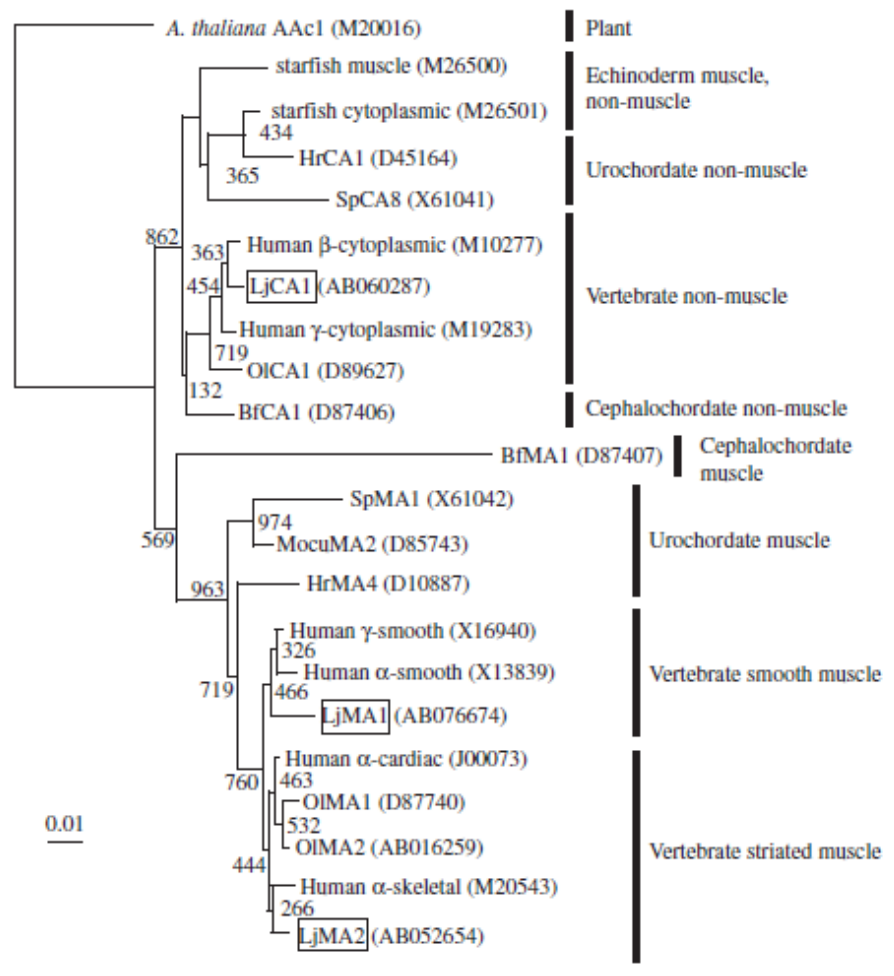

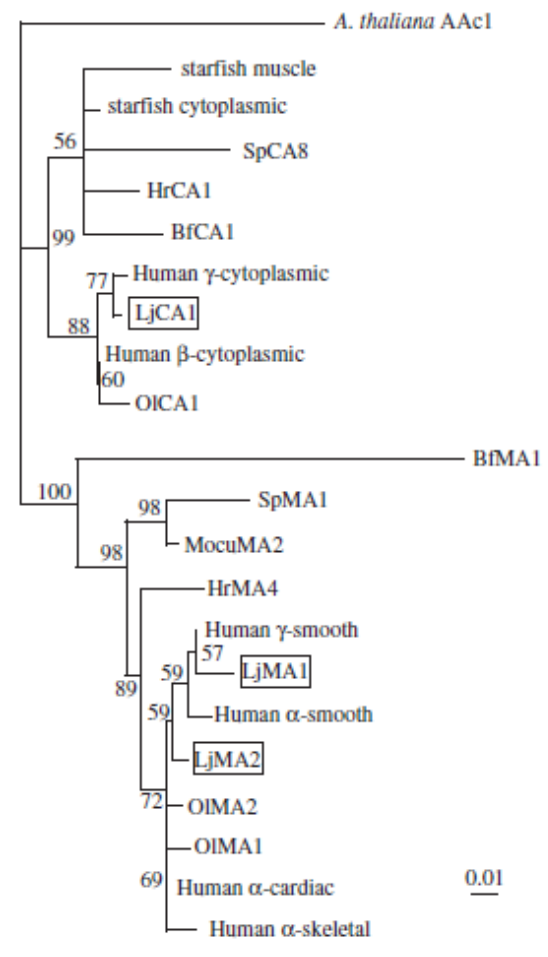

Fig. 2. Phylogenetic tree analysis of actin amino acid sequences. Lamprey L. japonicum actins are framed. The accession numbers are indicated in brackets. Some of the protein names are referred to by abbreviated forms of the genus and species; $\mathrm{Hr}$, ascidian Halocynthia roretzi; Sp, ascidian Styela plicata; Ci, ascidian Ciona intestinalis; Mocu, ascidian Molgula oculata; Bf, amphioxus Branchiostoma floridae; Lj, lamprey Lethenteron japonicum; Ol, teleost Olyzias latipes. Starfish muscle and cytoplasmic actins are those of Pisaster ochraceus. A. Phylogenetic tree constructed using the neighbor-joining method. Numbers indicate the bootstrap replicates of 1000 trials. B. Phylogenetic tree constructed using the maximum-likelihood method. Numbers indicate the quartet puzzling reliability values. 

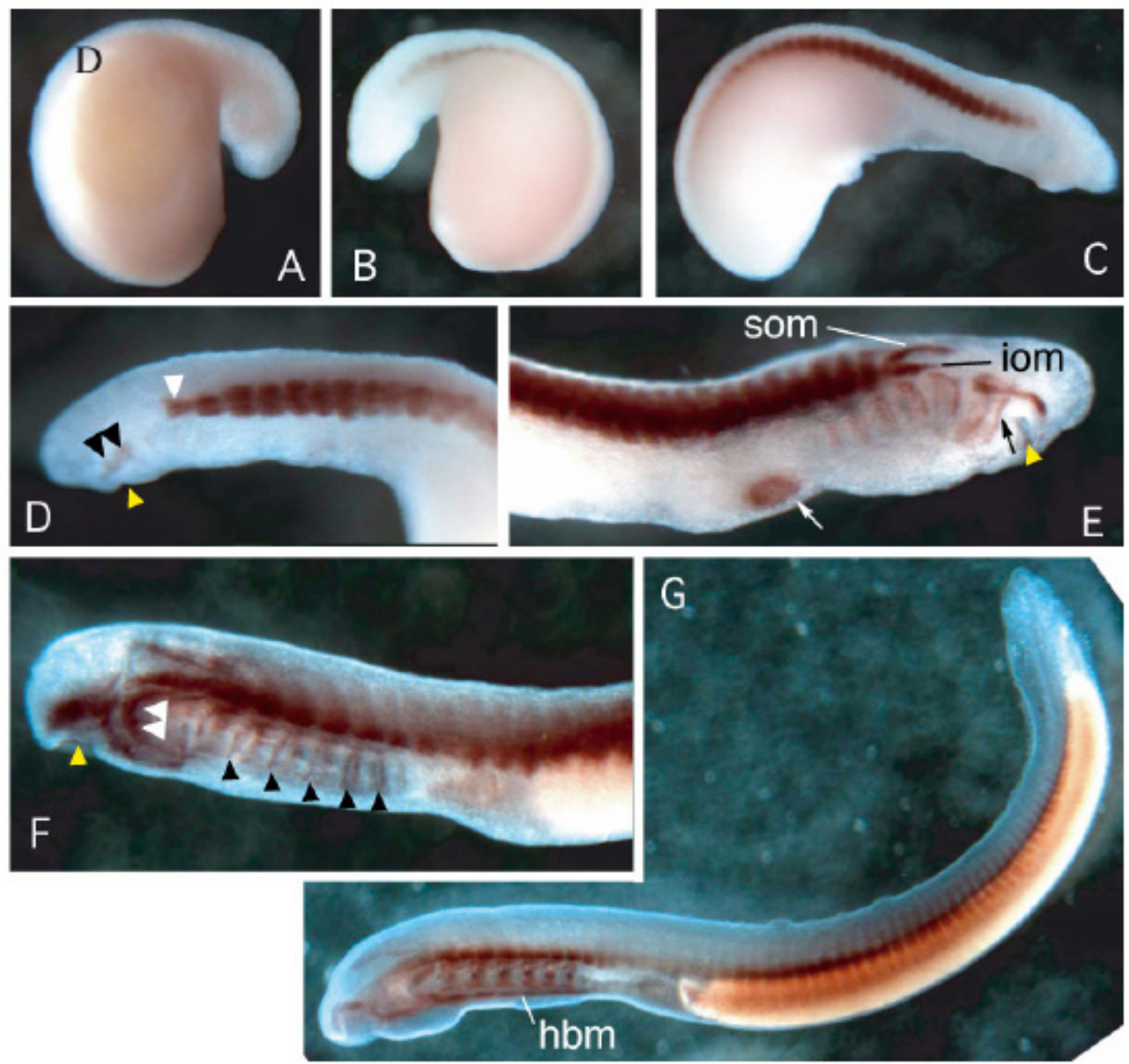

Fig. 3. Expression of lamprey actin gene LjMA2 during development. A. A stage 21.5 embryo, anterior is to the right. The dorsal side of the embryo is indicated by ' $\mathrm{D}$ ". No expression of LjMA2 is detected. B. A stage 22 embryo, anterior is to the left. LjMA2 expression begins at the anterior somites. C. A stage 23 embryo, anterior is to the right, dorsal is to the top. LjMA2 expression is expressed strongly in differentiating myotomes. D-F. The location of the mouth is indicated by an yellow arrowhead. D. A stage 24 embryo. The cells in the upper lip (black double arrowhead) start to express LjMA2. The most rostral myotome starts to split dorsoventrally (white arrowhead). E. At stage 25, LjMA2 expression in head mesoderm spreads to the lower lip (black arrow), the anterior branchial arches, and the cardiac muscle (white arrow). The most rostral myotomes split to form the supraand infraoptic muscles ("som" and "iom"). F. At stage 28, LjMA2 expression is detected in the upper and lower lip muscles, velum (white double arrowhead), and in each of the pharyngeal arches (black arrowheads). G. A stage 30 embryo. The hypobranchial muscle ("'hbm”) expresses LjMA2. 

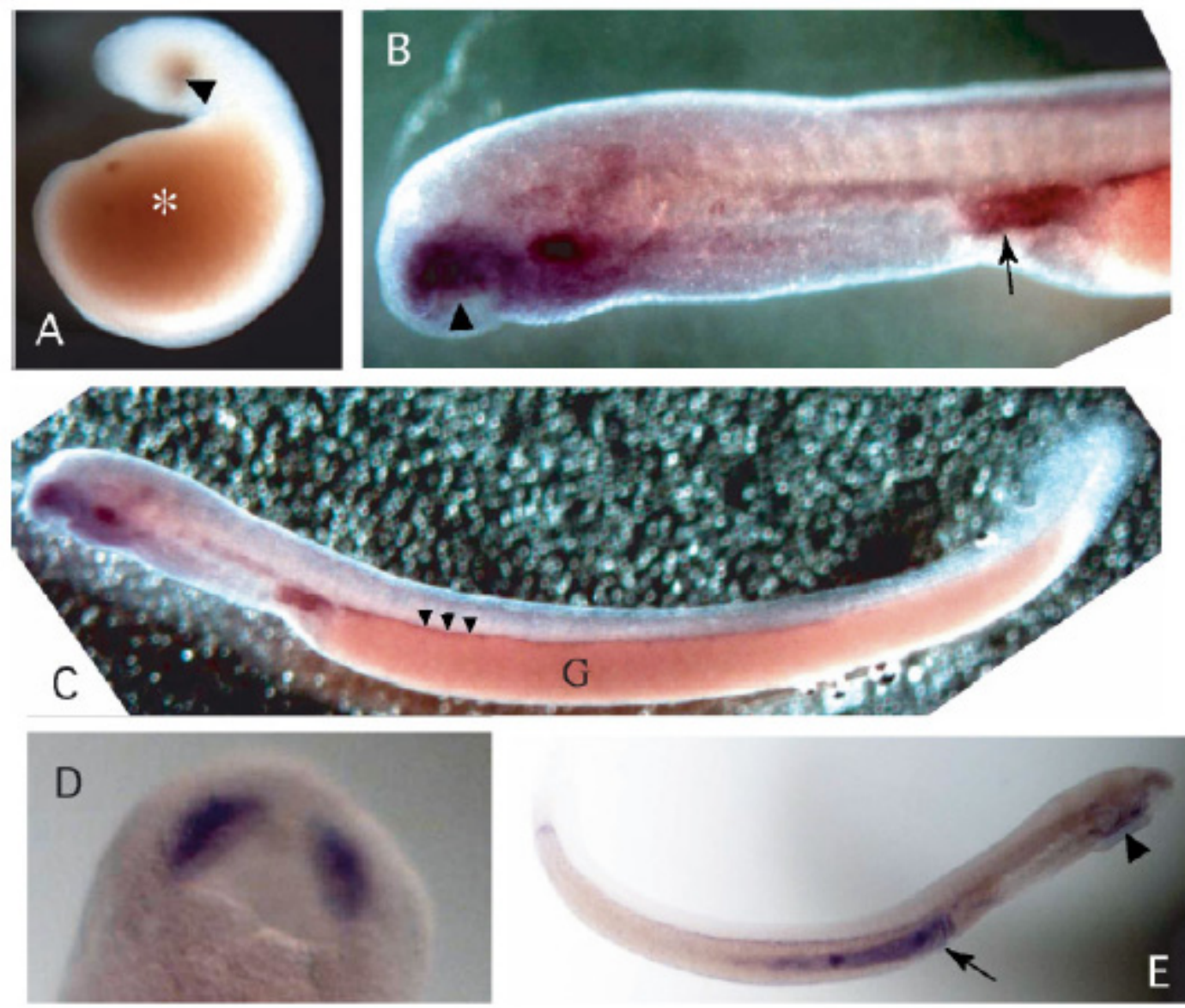

Fig. 4. Expression of lamprey actin gene LjMA1during development. A. LjMA1 expression starts at the cheek process (arrowhead) of stage 22 embryos. The dark area in the lateral trunk (asterisk) is a shadow caused by lighting for microscopic observation. B. The anterior part of a stage 28 embryo. LjMA1 expression is seen around the mouth (arrowhead) and in the digestive organ (arrow). C. A whole view of the embryo shown in B. The expression at the dorsal side of the gut (G) is indicated by black triangles. D, E. A stage 30 embryo. D. Ventral view of the most rostral part of the head. LjMA1 is expressed symmetrically in the upper lip. E. A stage 30 embryo, anterior is to the right. LjMA1 is expressed in the pharyngeal region (arrowhead) and the gut (arrow). 
A

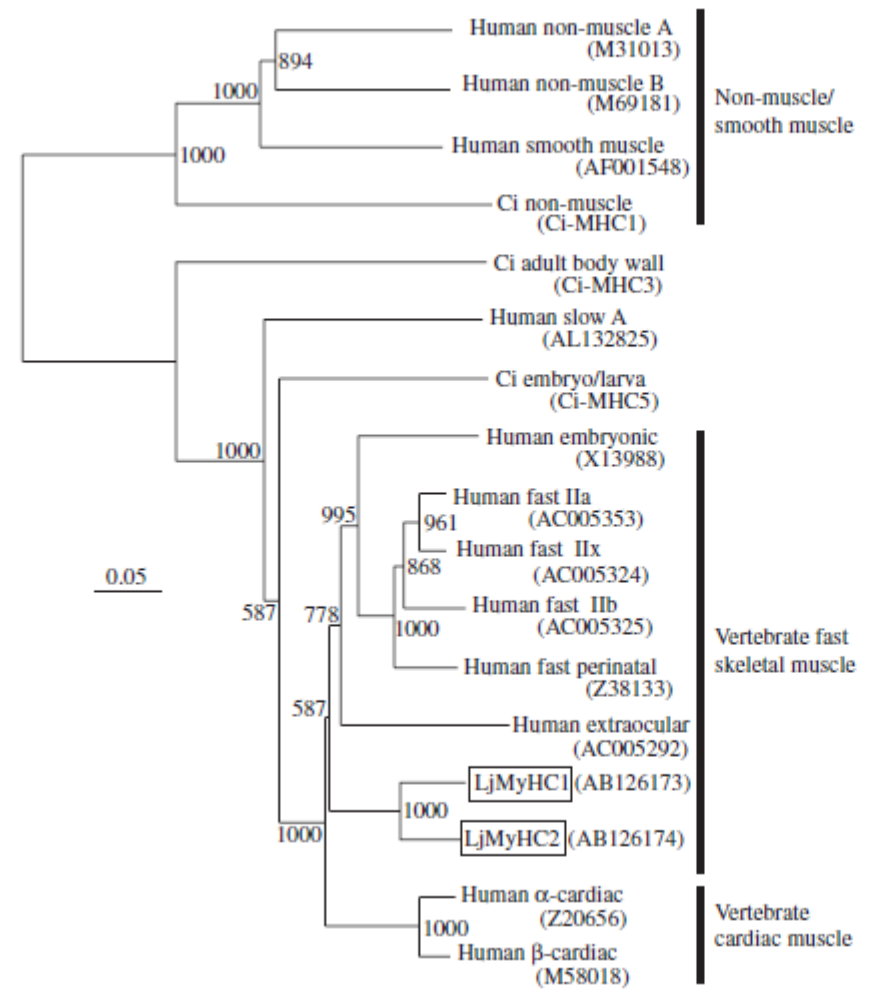

B

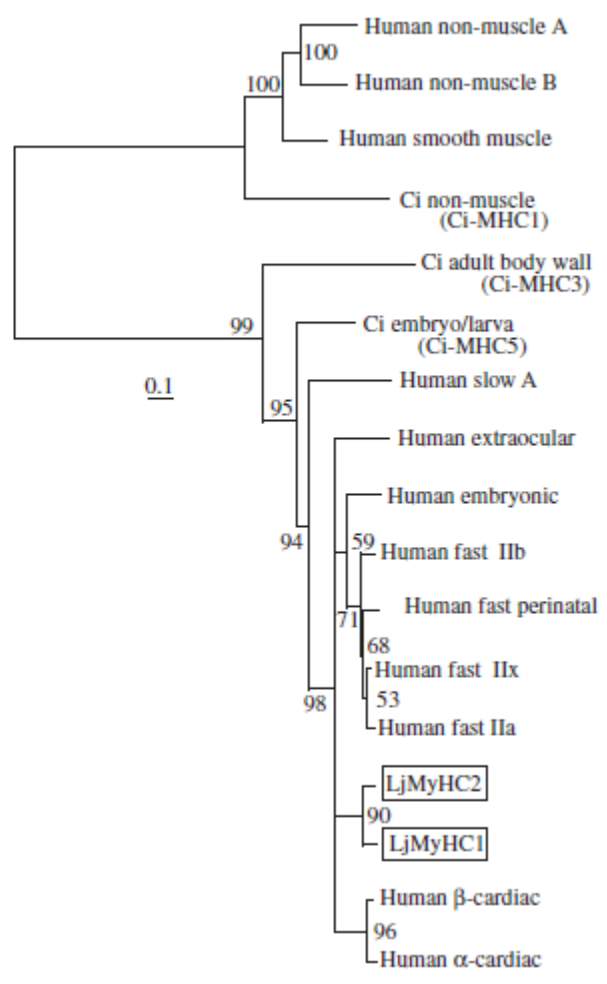

Fig. 5. Phylogenetic tree analysis of MyHC amino acid sequences. Lamprey L. japonicum MyHCs are framed. The references for C. intestinalis MyHCs are indicated in brackets by gene names in Chiba et al., (2003). A. Phylogenetic tree constructed using the neighbor-joining method. Numbers indicate the bootstrap replicates of 1000 trials. B. Phylogenetic tree constructed using the maximum-likelihood method. Numbers indicate the_quartet puzzling reliability values. 

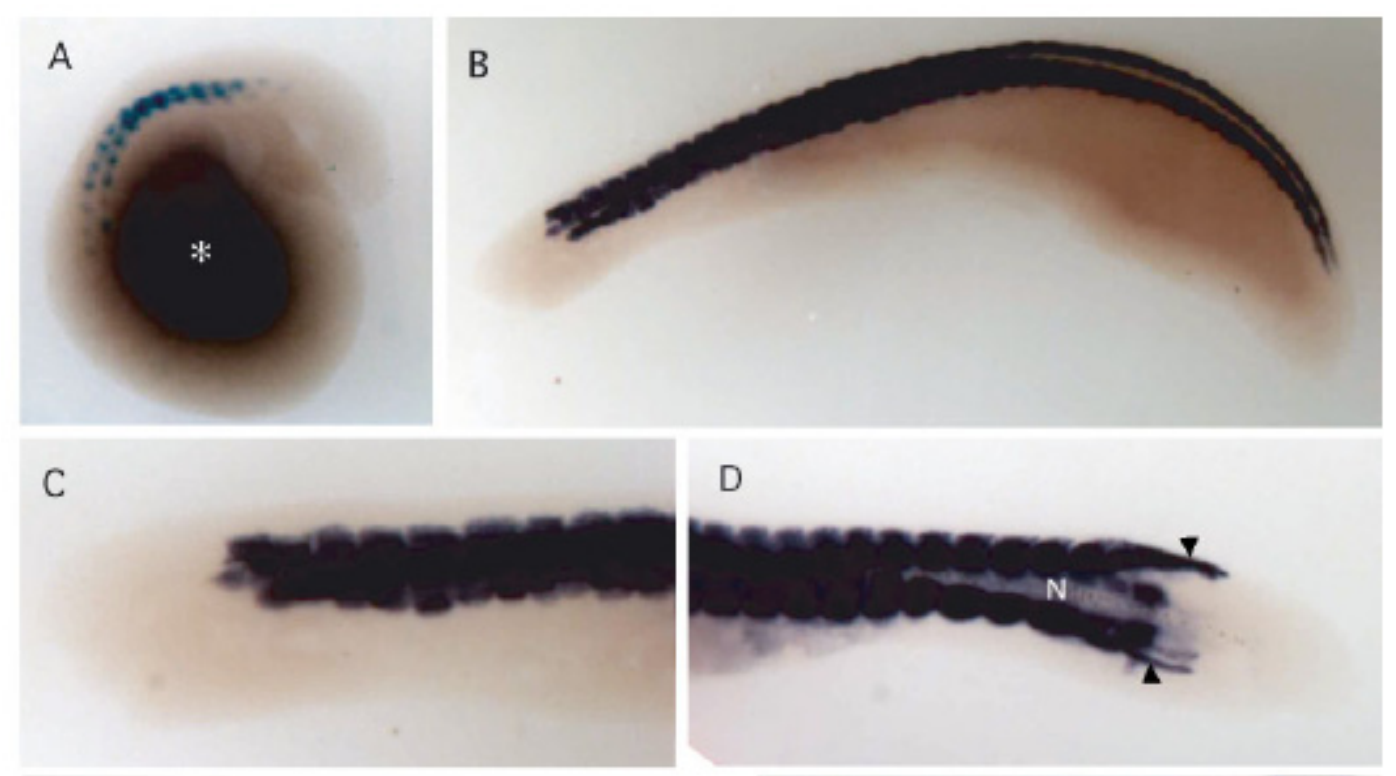

D
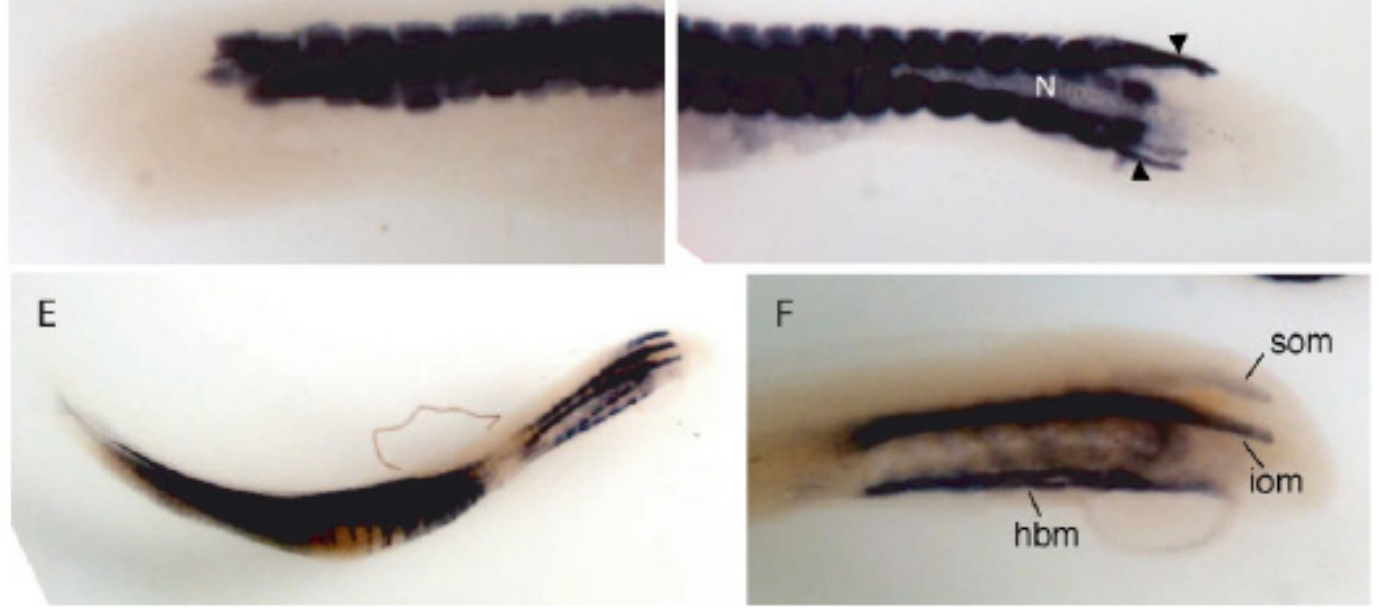

Fig. 6. Expression of the lamprey myosin heavy chain gene LjMyHC1 during development. A. A stage 23 embryo. LjMyHC1 expression starts in developing myotomes. In this embryo, two rows of myotomes on both sides of the body were visualized by the clearing step. The dark area in the lateral trunk (asterisk) is a shadow. B. A stage 24 embryo, anterior is to the left. Myotomal $\mathrm{LjMyHC1}$ expression extends caudally. C. Higher magnification of the head part of the embryo from B. No LjMyHC1 expression is observed rostral to the first somite, although some skeletal muscles have started to differentiate from head mesoderm at this stage. D. Dorsal view of the head part of a stage 29 embryo, anterior is to the right. Only the skeletal muscles derived from myotomes express LjMyHC1. The most rostral myotomes extend rostrally (arrowheads). N; notochord. E. A stage 30 embryo, anterior is to the right. LjMyHC1-positive trunk skeletal muscles undergo ventral extension. F. Head part of a stage 30 embryo. LjMyHC1-positive supra- and infraoptic muscles ("som" and "iom") and hypobranchial muscle (hbm) are all derived from myotomes, although located in the head. 

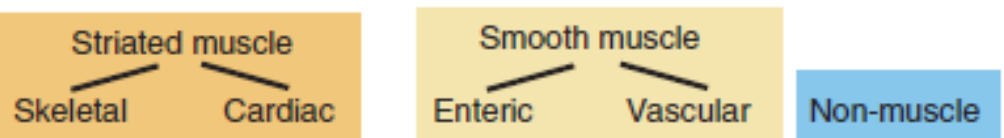

mammals/birds

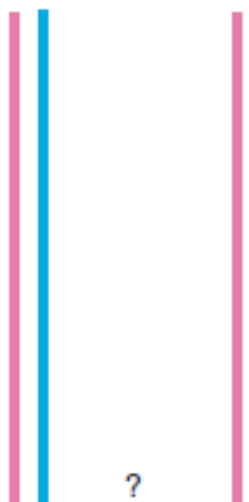

(a)

Vascular

Non-muscle

Reptiles

Amphibians

Bony fishes

Cartilageous fishes

Agnathans

Urochordates

Hemichordates/Echinoderms

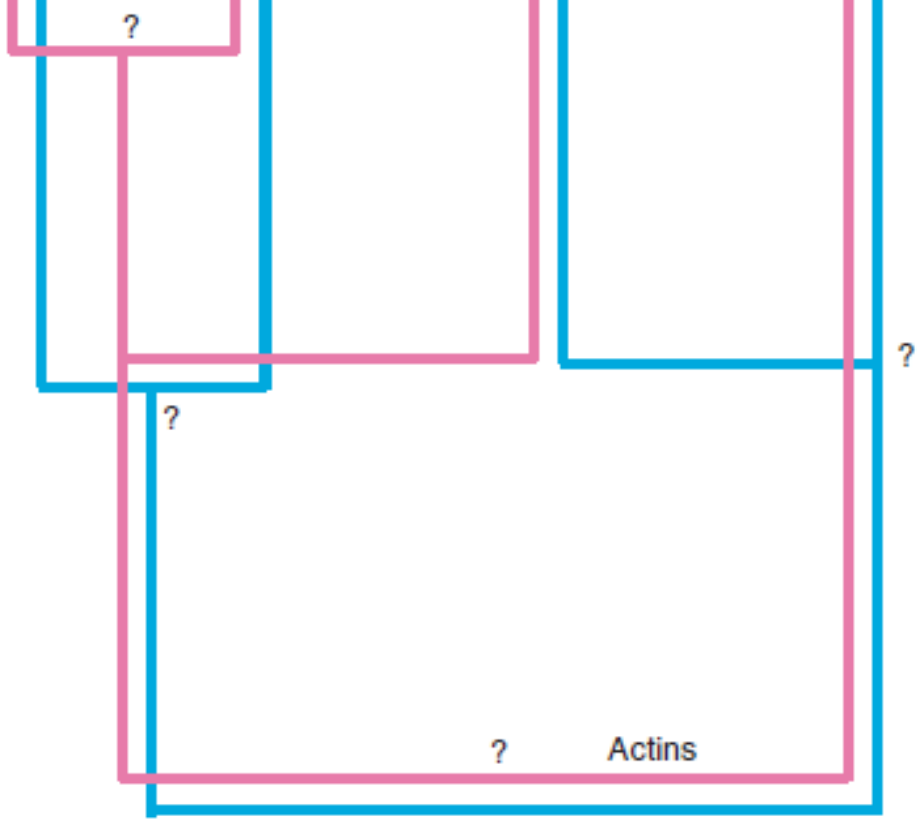

MyHCs

Fig. 7. Schematic evolutionary tree of vertebrate muscle actins and MyHCs based on the amino acid variations. Pink lines indicate divergence of actin proteins. Blue lines indicate divergence of MyHC proteins. Question marks indicate that the branching may be earlier than indicated, which has not been ruled out by this or previous studies. 